\title{
Distinct Roles for lonotropic and Metabotropic Glutamate Receptors in the Maturation of Excitatory Synapses
}

\author{
Stephen N. Gomperts, ${ }^{1}$ Reed Carroll, ${ }^{2}$ Robert C. Malenka, ${ }^{2,3}$ and Roger A. Nicoll ${ }^{1,3}$ \\ Departments of ${ }^{1}$ Cellular and Molecular Pharmacology, ${ }^{2}$ Psychiatry, and ${ }^{3}$ Physiology, University of California, San \\ Francisco, San Francisco, California 94143
}

\begin{abstract}
We used the single-cell culture preparation to study the role of activity in the development of glutamatergic synapses in vitro. Rat hippocampal cells grown in isolation on glial islands formed functional autaptic connections and continued to elaborate new synapses throughout the 2 week investigation, resulting in increases in both the evoked AMPA receptor (AMPAR) and NMDA receptor (NMDAR) components of the EPSC. Synaptogenesis was not prevented by chronic blockade of sodium channels or all of the known glutamate receptors. Analysis of miniature EPSCs revealed that AMPAR quantal size doubled over time in vitro whereas NMDAR quantal size remained constant. However, the proportion of synaptic responses mediated only by NMDARs increased over time in vitro. The increase in AMPAR quantal size was prevented by TTX and ionotropic
\end{abstract}

glutamate receptor antagonists, whereas the increase in the proportion of NMDAR-only synapses was prevented by metabotropic glutamate receptor antagonists. Notably, chronic NMDAR blockade incubation did not block the formation of the AMPAR EPSC, indicating that NMDAR-dependent plasticity is not necessary for the onset of AMPAR synaptic transmission in this system. We conclude that action potentials and ionotropic glutamate receptor activation are necessary for the developmental increase in AMPAR quantal size and that metabotropic glutamate receptor activation is required for the production of NMDAR-only synapses, but none of these is essential for synapse formation.

Key words: ionotropic; metabotropic; glutamate receptors; development; silent synapse; autapse
The formation of a synapse requires the development of both anatomical structure and physiological function. What signal(s) coordinates the assembly of the presynaptic and postsynaptic machinery required for synapse formation? An obvious possibility is that neurotransmitter released from synaptic vesicles at the growth cone or in the axon could activate neurotransmitter receptors in the postsynaptic cell membrane, thereby initiating the events required for synapse formation.

Recent immunocytochemical work on the formation of glycinergic synapses in spinal neuron cultures (Kirsch and Betz, 1998) supports this hypothesis. The authors found that the colocalization of glycine receptor clusters and presynaptic vesicle markers depended on action potentials, glycine receptor function, and L-type calcium channels. These data suggest that synapse formation depends on a signaling cascade in which the presynaptic terminal communicates its presence to the postsynaptic cell via the action potential-dependent release of neurotransmitter and

Received Oct. 1, 1999; revised Dec. 23, 1999; accepted Jan. 5, 2000.

S.N.G. is supported by the University of California, San Francisco, Medical Scientist Training Program Grant GM07618. R.A.N. is a member of the Keck Center for Integrative Neuroscience and the Silvio Conte Center for Neuroscience Research. R.C.M. was a member of the Center for Neurobiology and Psychiatry and the Center for the Neurobiology of Addiction. R.A.N. is supported by grants from the National Institutes of Health and Bristol-Myers Squibb Company. R.C.M. is supported by grants from the National Institutes of Health, the Human Frontier Science Program, and the McKnight Endowment Fund for Neuroscience. We thank members of the Nicoll and Malenka labs for their valuable input in the development of this work. We thank also H. Czerwonka for help in preparing this manuscript and C. Billante and S. Giller for preparing the autapse cultures.

Correspondence should be addressed to Dr. Roger A. Nicoll, Department of Cellular and Molecular Pharmacology, University of California, San Francisco, San Francisco, CA 94143-0450. E-mail: nicoll@phy.ucsf.edu.

Dr. Carroll's and Dr. Malenka's present address: The Nancy Pritzker Laboratory, Department of Psychiatry and Behavioral Sciences, Stanford University School of Medicine, Stanford, CA 94305.

Copyright (C) 2000 Society for Neuroscience $0270-6474 / 00 / 202229-09 \$ 15.00 / 0$ subsequent activation of postsynaptic receptors. It is important to note, however, that this mechanism does not apply to all synapses. In particular, the formation of the neuromuscular junction does not depend on action potentials (Davey and Cohen, 1986) or the activation of endplate acetylcholine receptors (Cohen, 1972).

Excitatory synapses using the neurotransmitter glutamate contain both ionotropic and metabotropic glutamate receptors. The ionotropic glutamate receptor family can be further divided into the NMDA receptor (NMDAR) and the non-NMDA receptor [AMPA receptor (AMPAR) and kainate receptor] subfamilies. Previous work has shown that AMPAR and NMDAR accumulation at the synapse continues under action potential blockade (Craig et al., 1994; O'Brien et al., 1997) and under AMPAR and NMDAR blockade (O'Brien et al., 1997; Rao et al., 1998; Liao et al., 1999). However, the possible involvement of metabotropic or kainate subtypes of glutamate receptors in synapse formation has not been addressed.

An additional complexity of synaptogenesis of excitatory synapses is that glutamatergic synapses vary in their complement of receptors. Only a subset have kainate receptors (Lerma et al., 1997), and although many synapses have both AMPARs and NMDARs, some have only NMDARs (NMDAR-only synapses) (Rao and Craig, 1997; Gomperts et al., 1998; Nusser et al., 1998; Liao et al., 1999; Petralia et al., 1999; Takumi et al., 1999). Furthermore, in a number of preparations synaptic responses mediated only by NMDARs are more prevalent earlier in development (Durand et al., 1996; Wu et al., 1996; Isaac et al., 1997; Hsia et al., 1998; Rumpel et al., 1998). Recent anatomical data suggest that these so-called "silent" synapses derive from an initial developmental absence of AMPARs at synapses (Liao et al., 1999; Petralia et al., 1999), although this has not been observed in all preparations (Rao et al., 1998). These data, coupled 
with the observation that induction of long-term potentiation can reveal AMPAR EPSCs in recordings of NMDAR-only synaptic responses (Isaac et al., 1995; Liao et al., 1995), suggest that the developmental recruitment of AMPARs to synapses may involve a Hebbian, NMDAR- and activity-dependent process.

To study the formation and maturation of synapses and the role of activity in these processes, we examined single-neuron cultures (Segal and Furshpan, 1990). This preparation permits sampling of all the synaptic inputs onto (and outputs from) recorded cells while providing access to the quantal parameters of individual synapses. Furthermore, because in single-cell cultures all synaptic events mediated by AMPARs are recorded, this preparation permits examination of NMDAR-only synaptic responses, stripped of the possible contribution of spillover of glutamate from adjacent synapses (Gomperts et al., 1998). Our results identify both activity-dependent and activity-independent processes that contribute to the formation and maturation of glutamatergic synapses.

\section{MATERIALS AND METHODS}

Tissue culture preparation. Microdot cultures were prepared from hippocampal neurons of the CA1 and CA 3 regions of the hippocampus of postnatal day 0 Sprague Dawley rat pups. The dentate gyrus was grossly dissected away, and cells from the remaining tissue were prepared as described by Tong and Jahr (1994), except that papain was not used and B27 (Life Technologies, Gaithersburg, MD) was supplemented. Because some proteases can selectively digest surface NMDARs (Allen et al., 1988; Murase et al., 1989), we avoided enzymatic digestion of hippocampal tissue. The growth medium was exchanged fully $1 \mathrm{~d}$ after plating and weekly in part thereafter. Autaptic recordings were obtained from isolated neurons grown on collagen and poly-D-lysine microdots. The number of isolated cells did not change appreciably between 3-5 and 11-14 d in vitro (div) ( $n=3$ culture preparations; young, $n=199$ isolated cells; old, $n=186$ ). Pharmacological antagonists were added at the time of plating in the following concentrations: D-aminophosphonovalerate (APV), $100 \mu \mathrm{M}$; 6-nitro-7-sulfamoylbenzo(f)quinoxaline-2,3-dione (NBQX) disodium salt, $50 \mu \mathrm{M}$; methyl-4-carboxyphenylglycine (MCPG), 1-2 mM; TTX, $1 \mu \mathrm{M}$; and 4-aminopyridine (4-AP), $50 \mu \mathrm{M}$. They were replaced at these concentrations when the medium was exchanged the day after plating and were renewed daily at the following concentrations: APV, $100 \mu \mathrm{M}$; NBQX disodium salt, $5 \mu \mathrm{M}$; MCPG, $1 \mathrm{mM}$; TTX, $1 \mu \mathrm{M}$; and 4-AP, $25 \mu \mathrm{M}$. Cells were fed at 1 week, with replacement of one-half of the cell medium. APV, NBQX disodium salt, and MCPG were from Tocris Cookson. TTX was purchased from Calbiochem (La Jolla, CA).

Whole-cell recording. Recordings were made at room temperature from 2- to 15-d-old isolated autaptic neurons, using an Axopatch-1D amplifier with low-resistance patch pipettes (2-5 M $\Omega$ ). Pipette solutions contained $122.5 \mathrm{~mm}$ K-gluconate, $8 \mathrm{~mm} \mathrm{NaCl}, 10 \mathrm{~mm}$ HEPES, $0.2 \mathrm{~mm}$ EGTA, $2 \mathrm{~mm}$ MgATP, $0.3 \mathrm{~mm} \mathrm{NaGTP,} 20 \mathrm{~mm} \mathrm{~K}_{2}$-creatine phosphate, and $50 \mathrm{U} / \mathrm{ml}$ phosphocreatine kinase, adjusted to $\mathrm{pH} 7.4$ with $\mathrm{KOH}$. The extracellular solution was magnesium-free and contained $140 \mathrm{~mm} \mathrm{NaCl}, 3.5 \mathrm{~mm} \mathrm{KCl}$, $10 \mathrm{~mm}$ HEPES, $20 \mathrm{~mm}$ glucose, 0.3 or $3 \mathrm{~mm} \mathrm{CaCl}_{2}$, and $20 \mu \mathrm{M}$ glycine, adjusted to $\mathrm{pH} 7.3$ with $\mathrm{NaOH}$. TTX $(1 \mu \mathrm{M})$ did not alter the amplitude or frequency of spontaneous events recorded in isolated autaptic cells ( $n=3$; data not shown) and, therefore, was generally not used for the acquisition of miniature EPSCs.

Cells were held at $-70 \mathrm{mV}$ and were stimulated every $10-15 \mathrm{sec}$ with a 2.0-2.5 msec $70 \mathrm{mV}$ depolarizing current pulse. AMPAR-mediated currents were isolated with the addition of 100-150 $\mu \mathrm{M}$ APV; NMDARmediated currents were isolated by the addition of 5-7.5 $\mu \mathrm{M} \mathrm{NBQX}$ disodium salt. Synaptic currents were completely abolished with the addition of both APV and NBQX disodium salt. Some AMPAR/ NMDAR amplitude ratios were derived in $0.3 \mathrm{~mm} \mathrm{Ca}$, but this did not affect the mean AMPAR/NMDAR ratio, and 0.3 and $3 \mathrm{~mm} \mathrm{Ca}$ data were pooled. Series resistance ranged from 8 to $24 \mathrm{M} \Omega$ and was compensated $(80 \%)$ in all experiments. The series and input resistances were monitored throughout each experiment with a $3 \mathrm{mV}$ calibration pulse given $40 \mathrm{msec}$ before each stimulation. Junction potentials were corrected and were $\sim 10 \mathrm{mV}$. Evoked EPSCs were acquired and analyzed on-line using custom software (D. Selig). Currents were low-pass filtered at $2 \mathrm{kHz}$ and digitally sampled at $5 \mathrm{kHz}$.
Analysis of EPSCs and measurement of the receptor colocalization index. The AMPAR and NMDAR amplitudes for the evoked responses were taken either from the individual components or from the AMPAR and dual-component EPSCs and were measured from the average of 10-30 traces. Miniature EPSCs (mEPSCs) were acquired using Axoscope (Axon Instruments) and were analyzed using Mini (J. H. Steinbach) and Quanta (S. Borges). The threshold mEPSC amplitude was set at $5 \mathrm{pA}$, and events were collected in each pharmacological condition. The AMPAR quantal amplitude was measured from mEPSCs recorded in APV. The quantal amplitude of the NMDAR component of dual-component mEPSCs was measured by averaging together dual-component mEPSCs recorded without receptor antagonists, subtracting the scaled, averaged AMPAR mEPSC to derive the NMDAR mEPSC, and scaling the NMDAR quantal amplitude by the ratio of the AMPAR quantal amplitudes derived with and without APV. Although scaling was often necessary, because the smallest AMPAR events can get buried in spontaneous NMDAR activity, this is unlikely to be a source of error (Gomperts et al., 1998). The ratio of the respective EPSC amplitude to the mEPSC amplitude represents the quantal content of the AMPAR response $(A)$ and the NMDAR response $(N)$. The fraction of NMDAR-only synapses, estimated as the fraction of NMDAR synapses in excess of AMPAR synapses, can be calculated as a linear quantity, the receptor colocalization index [r.c.i. $=(N-A) /(N+A)]$, which ranges from all NMDARonly synapses (a value of 1 ) to all AMPAR-only synapses (a value of -1). The r.c.i. was calculated for each cell in each condition to derive mean r.c.i. values. However, because of the difficulty in sustaining recordings in young cells, the r.c.i. of young cells was calculated from the mean quantal contents of all cells. This method gave the same r.c.i. value for old cells as that of the within-cell analysis. It is possible that some of the variance in our data set derives from drift in the properties of the cultures over multiple culture preparations. To assess this contribution of variance, we examined the variance across preparations for our experimental measurements with the largest number of samples, evoked AMPAR and NMDAR amplitudes. For all conditions, the ANOVA showed no significant differences in values between preparations (data not shown), and so we conclude that variability across cultures is not a major contributor to our measurements.

Immunocytochemistry. Cells were grown as described for electrophysiological experiments. AMPARs were detected using a rabbit polyclonal antibody to the $\mathrm{N}$ terminal of the glutamate receptor 1 (GluR1) subunit (Oncogene). Live cells were incubated in antibody $(5 \mu \mathrm{g} / \mathrm{ml})$ for $20 \mathrm{~min}$ at $37^{\circ} \mathrm{C}$ in the conditioned cell media. Cells were then fixed in methanol for $15 \mathrm{~min}$ at $-20^{\circ} \mathrm{C}$, air dried, and blocked with $10 \%$ BSA in TBS. NMDARs were detected using monoclonal antibodies to the intracellular loop between transmembrane regions III and IV of the NR1 subunit (PharMingen, San Diego, CA). The anti-NR1 antibody was applied overnight at $4^{\circ} \mathrm{C}$. After several washes, fluorochrome-conjugated [FITC for GluR1 and indocarbocyanine (Cy3) for NR1] donkey anti-mouse or anti-rabbit secondary antibodies (Jackson ImmunoResearch, West Grove, PA) were concurrently applied to cells for $1 \mathrm{hr}$ at room temperature. After several washes, slips were mounted in Vectashield (Vector Laboratories, Burlingame, CA) mounting medium. Identification of clusters of glutamate receptors was accomplished with dual-color microscopy using a Nikon $60 \times$ objective (numerical aperture, 1.4) and standard fluoroscein and Cy3 filter sets (Omega). Fluorescent images of microscopic fields containing labeled neurons were acquired using a cooled CCD camera (Princeton Instruments, Inc.). Fields of GluR1-stained cells were chosen randomly, and images from both fluorescent channels were successively acquired from a single focal plane. Acquired images were normalized to maximal contrast, overlayed, and analyzed using IPLab Spectrum software (Signal Analytics). For display in figures, monochrome and merged color images were digitally processed using Adobe Photoshop. For quantitative analysis, images were analyzed in a blinded manner such that the investigator had no knowledge of the treatment history of the cells being analyzed. Colocalization was determined by examination of the overlayed receptor-stained images. Out-of-focus and extended nondiscreet regions of staining were not included in the quantitation. For the immunocytochemical experiment, $n$ refers to the number of cells analyzed.

Data analysis. Results are presented as means \pm SE. Data were compared statistically using the Student's $t$ test, and significance was defined at $p<0.05$. 

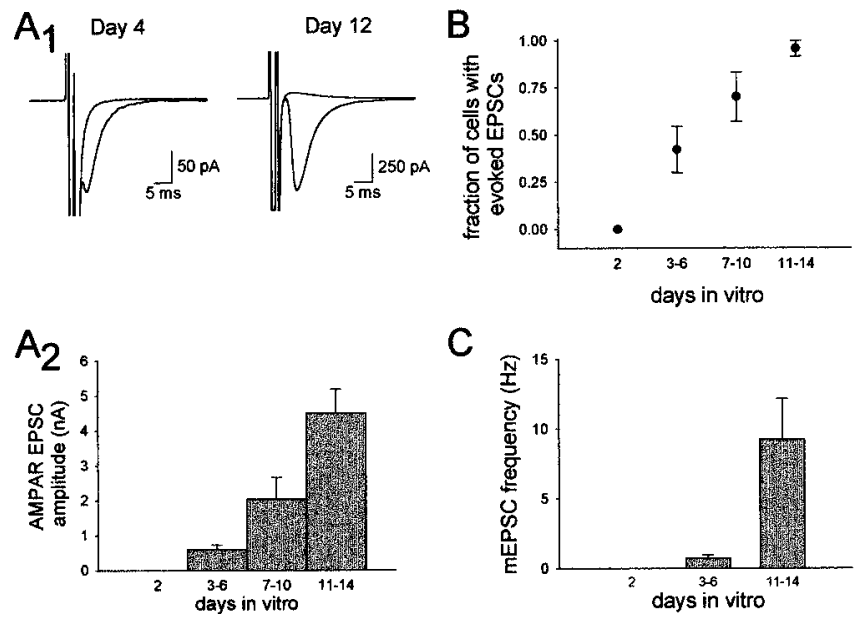

Figure 1. Synaptogenesis is ongoing in autaptic cells. $A$, The evoked AMPAR EPSC increases with time in vitro. $A_{l}$, Representative average traces from 4 and 12 div cells of AMPAR EPSCs (isolated with APV) superimposed on the action potential artifact (isolated with APV and NBQX) are shown. Note the different calibrations. Traces are the average of 15 responses in either $100 \mu \mathrm{M}$ APV or $100 \mu \mathrm{M}$ APV with $5 \mu \mathrm{M}$ NBQX. $A_{2}$, In the subpopulation of cells that show evoked responses, the evoked AMPAR EPSC increases developmentally $[\mathrm{n}=33$ (3-6 div); $n=6$ (7-10 $\operatorname{div}) ; n=36(11-14$ div $)]$. $B$, The proportion of neurons that have evoked responses increases with time in vitro. Neurons were identified by the presence of action potentials and were tested for AMPAR and NMDAR EPSCs. Data in each age group were pooled by day $[n=4(2 \mathrm{div}) ; n=83$ (3-6 div); $n=14$ (7-10 div); $n=16$ (11-14 div)]. $C$, The frequency of miniature AMPAR EPSCs undergoes a marked developmental increase $[n=4$ (2 div); $n=7$ (3-6 div); $n=19$ (11-14 div)].

\section{RESULTS}

\section{Natural history of synapse development in autaptic hippocampal cells}

At birth, no synaptic responses can be observed, and few synapses are evident anatomically in the hippocampus of Sprague Dawley rats (Steward and Falk, 1991; Durand et al., 1996). We therefore dissociated and cultured cells from the CA1 and CA3 hippocampal regions of newborn Sprague Dawley rats to examine de novo synaptogenesis. Rat hippocampal cells grown in isolation on glial islands formed functional autaptic connections and continued to elaborate synapses throughout our 2 week period of study. Although the action potential was present at 2 div, no EPSCs were observable at this time (Fig. $1 B$ ). EPSCs were first observed in a subset of cells at 3 div. Although it is possible that the absence of evoked responses might originate in a failure of the action potential generated at the cell body to propagate to the terminals (Kimura et al., 1997), there were virtually no spontaneous events in cells that lacked evoked responses ( $n=57$ of 58). Addition of a hyperosmolar solution (100 mOsm sucrose) to cells lacking evoked and spontaneous AMPAR-mediated events also failed to generate detectable EPSCs $(n=4)$ (Basarsky et al., 1994). Thus, functional synapses appeared to be absent in these young cells.

To determine whether the lack of evoked and miniature EPSCs in these cells was caused by the absence of functional AMPARs, we applied AMPA; in every cell tested $(n=7)$ AMPA elicited inward currents. However, addition of cyclothiazide, which increases the size and duration of AMPAR EPSCs, did not reveal AMPAR EPSCs in cells that lacked them under normal recording conditions $(n=4)$. These data indicate that AMPARs are present and functional in the postsynaptic cell membrane but are not sensing released neurotransmitter. The defect in synaptic

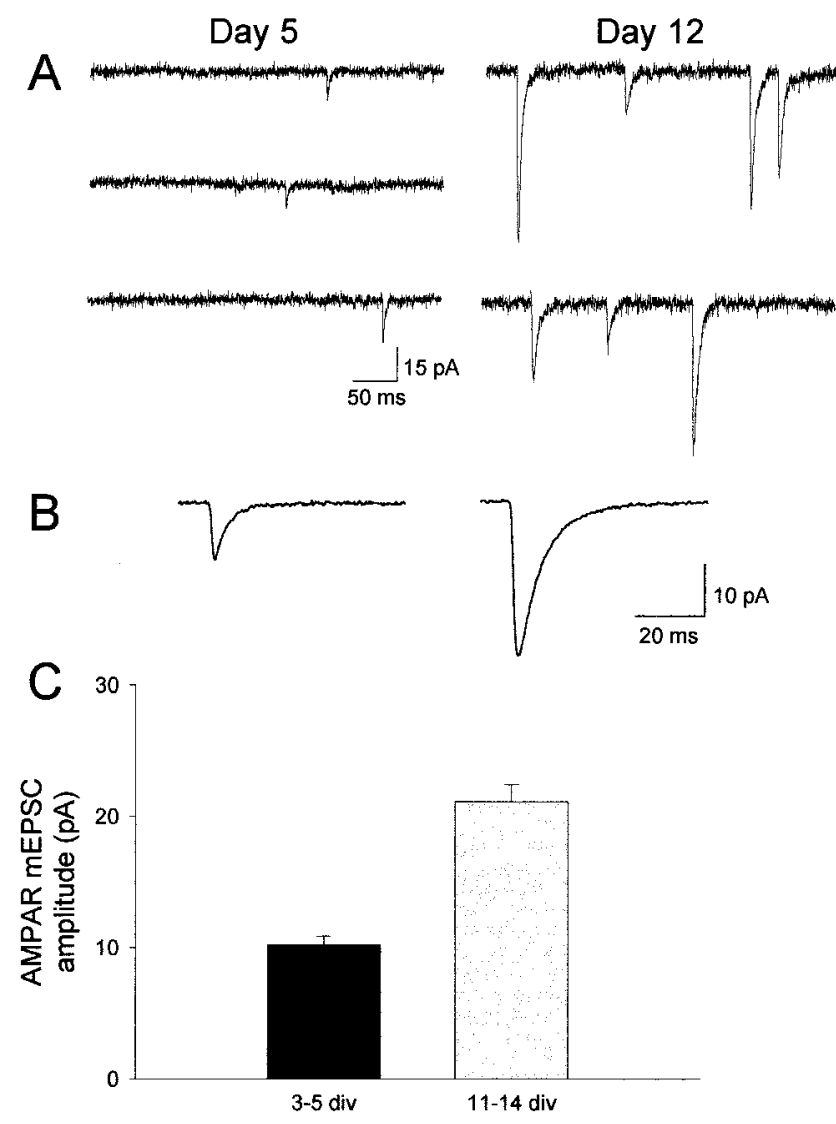

Figure 2. AMPAR quantal size increases over development. $A$, Representative recordings in $100 \mu \mathrm{M}$ APV from a day 5 cell and a day 12 cell are shown. $B$, Representative averaged traces of spontaneous events derived in APV from the day 5 cell (49 traces; left) and the day 12 cell (97 traces; right) are shown. $C$, The mean AMPAR mEPSC amplitude is larger in older cells $[n=8$ (3-5 div); $n=18$ (11-14 div); $p<0.01]$.

responsiveness in these young cells could derive from a lack of anatomical synapses, from the absence of the vesicular release of glutamate, or from an absence of receptor clustering at synapses.

From 3 to 15 div, the pharmacologically isolated AMPA receptor-mediated EPSCs increased approximately eightfold (Fig. $1 A$ ). The fraction of cells that had evoked synaptic responses increased concomitantly (Fig. $1 B$ ). In agreement with an increase in the number of functional synapses, the frequency of mEPSCs increased $\sim 10$-fold over this period, from $0.7 \pm 0.3 \mathrm{~Hz}(n=7)$ to $9.2 \pm 2.9 \mathrm{~Hz}(n=19)$ (Fig. $1 C)$.

To assess whether changes in the quantal properties of synapses contribute to the increase in the evoked AMPAR EPSCs, we examined AMPAR quantal size. As shown in Figure 2, AMPAR quantal size doubled over this period (young, $n=8$; mean $=$ $10.2 \pm 0.7 \mathrm{pA}$; old, $n=18$; mean $=21.1 \pm 1.3 \mathrm{pA} ; p<0.01)$. This twofold increase can only account for a small fraction of the overall growth in evoked EPSC amplitude. The quantal content of the AMPAR EPSC, defined as the product of $N$ and $p$ and calculated as the ratio of mean EPSC amplitude to mean quantal amplitude, also increased over development $(p<0.01)$. Because the probability of release, as assessed by measuring paired-pulse facilitation of the AMPAR EPSC (Manabe et al., 1993), did not increase developmentally (young, $n=5$; old, $n=4$; data not shown), a continuous, ongoing proliferation of synapses throughout the time in culture presumably underlies these observations 
(Benson and Cohen, 1996). Thus, during development, both an increase in synapse number and an increase in quantal size contribute to the observed increase in the evoked AMPAR EPSC.

It has been suggested that the AMPAR and NMDAR synaptic components of glutamatergic synapses develop on different timescales, with the NMDAR EPSC preceding the AMPAR EPSC developmentally (Durand et al., 1996; Wu et al., 1996; Isaac et al., 1997; Hsia et al., 1998; Rumpel et al., 1998; Liao et al., 1999; but see Rao et al., 1998). We therefore turned our attention to the development of the NMDAR-mediated component of synaptic responses. Even at the earliest time points, the evoked responses in the vast majority of cells had both NMDAR- and AMPARmediated currents ( $n=29$ of 31 cells). Like the AMPAR EPSC, the NMDAR EPSC increased dramatically from 3-5 to 11-14 div (Fig. 3A). However, NMDAR quantal size, measured from the dual-component mEPSC, was stable over development (young, $n=5$; mean $=4.8 \pm 1.3 \mathrm{pA}$; old, $n=13$; mean $=5.3 \pm 0.4 \mathrm{pA}$; $p>0.05$ ) (Fig. $3 B$ ). This suggests that synaptogenesis accounts entirely for the increase in NMDAR EPSC amplitude.

To assess whether the addition of NMDARs and AMPARs to synapses occurs in parallel in isolated cells, we first examined the AMPAR/NMDAR amplitude ratio. Despite the selective doubling of AMPAR quantal size from 3-5 to 11-14 div, the evoked AMPAR/NMDAR amplitude ratio actually decreased approximately twofold, from $4.4 \pm 0.5$ to $2.3 \pm 0.2$ (young, $n=36$; old, $n=46 ; p<0.01$ ) (Fig. $3 C$ ). To determine whether this increase in the proportion of NMDAR current derived from a change in the fraction of synapses that have only NMDARs (NMDAR-only synapses), we calculated the fraction of NMDAR-only synapses from the difference in quantal contents (mean EPSC size/mean mEPSC size) of AMPAR and NMDAR EPSCs. This technique is based on the idea that the population of NMDAR-only events will contribute selectively to the evoked NMDAR component (Gomperts et al., 1998). We express this estimate in terms of a receptor colocalization index (r.c.i.), a linear function that ranges from all NMDAR-only synapses (a measured value of 1) to all AMPAR-only synapses (a measured value of -1 ; see Materials and Methods). Such a term takes into account not just NMDARonly synapses but also the possibility of a population of AMPARonly synapses (Bekkers and Stevens, 1989), which would lead to an underestimate of the calculated proportion of NMDAR-only synapses. This analysis demonstrated a significant increase in the proportion of NMDAR-only synaptic events of older cells (old, $n=9$; r.c.i. $=0.30 \pm 0.07$; young, $n=5$; r.c.i. $=-0.35 \pm 0.21 ; p<$ 0.01 ) (Fig. $3 D$ ). The r.c.i. of the old group of cells is significantly different from zero $(p<0.01)$, suggesting a significant population of NMDAR-only synapses in these cells. We conclude from this set of experiments that isolated autaptic cells continuously proliferate synapses that have an NMDAR component, that NMDAR-only synapses represent an increasing proportion of synapses over time, and that NMDAR quantal size does not change over our time of observation.

\section{Testing a role for activity in synapse formation}

The formation of glycinergic synapses in spinal cultures is activity dependent (Kirsch and Betz, 1998). We therefore set out to assess a role for activity in the formation of glutamatergic synapses. Because of the literature implicating the NMDAR in the activitydependent production of AMPAR EPSCs (Isaac et al., 1995; Liao et al., 1995; Wu et al., 1996), we first grew our cultures in the NMDAR antagonist APV (100 $\mu \mathrm{M}$, replenished daily). Neurons

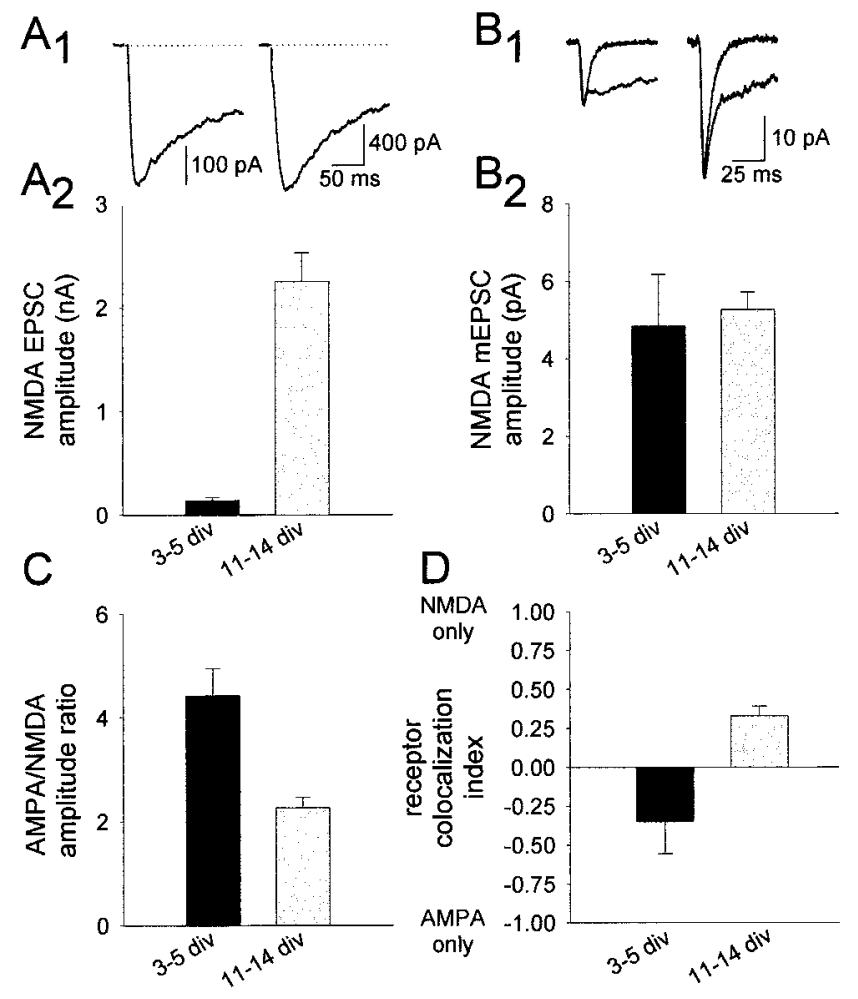

Figure 3. NMDAR EPSCs also increase over development. $A$, The evoked NMDAR EPSC increases developmentally. $A_{1}$, Representative evoked NMDAR EPSCs from a day 4 cell (left) and a 12 day cell (right) cell demonstrate the large developmental increase in amplitude. Each trace is the average of 10 events. Note the different calibrations. $A_{2}$, This increase was consistent for the population of cells examined $[n=34$ (3-5 div); $n=20$ (11-14 div)]. $B$, The quantal amplitude of the NMDAR component of dual-component mEPSCs is stable over the developmental period. $B_{1}$, Averaged representative traces from a day 4 (left) and a day 12 (right) cell demonstrate that the sizable increase in AMPAR quantal amplitude is not accompanied by a change in the NMDAR component of mEPSCs. The isolated AMPAR mEPSC, derived in APV, is shown superimposed and scaled to the peak of the dual-component event. One hundred eleven dual-component events and 27 events derived in APV comprise the average traces of the young cell. Forty-four dual-component and 38 APV events comprise the average traces of the old cell. $B_{2}$, Average NMDAR quantal amplitudes from 5 young and 13 old cells are similar. $C$, The AMPAR/NMDAR amplitude ratio of evoked EPSCs decreases twofold developmentally. AMPAR and NMDAR components were isolated pharmacologically and collected sequentially as the average of 10-30 traces each from 37 young and 46 old cells $(p<0.01)$. D, The fraction of NMDAR-only synapses increases over development in isolated cells. Differences in NMDAR and AMPAR quantal contents calculated from a comparison of evoked and spontaneous dual-component events are used to make this calculation. The results are displayed in terms of a linear function, the r.c.i., which ranges from all NMDAR-only synapses to all AMPAR-only synapses. Although the young cell r.c.i. is not significantly different from zero, the old cell r.c.i. is significantly different from zero (young, $n=5$; old, $n=9$; young vs old, $p<0.01$; old vs zero, $p<0.01$ ).

grown in APV, however, continued to demonstrate robust AMPAR and NMDAR EPSCs after the washout of APV (see Fig. 4). We therefore considered the possibility that glutamate signaling via other glutamate receptors may coordinate synapse formation. To block all glutamate receptors, we grew cells from their time of plating in ionotropic and metabotropic glutamate receptor antagonists: the NMDAR antagonist APV, the non-NMDAR antagonist NBQX, and the metabotropic glutamate receptor antagonist MCPG, replenished daily. Both AMPAR- and NMDAR- 


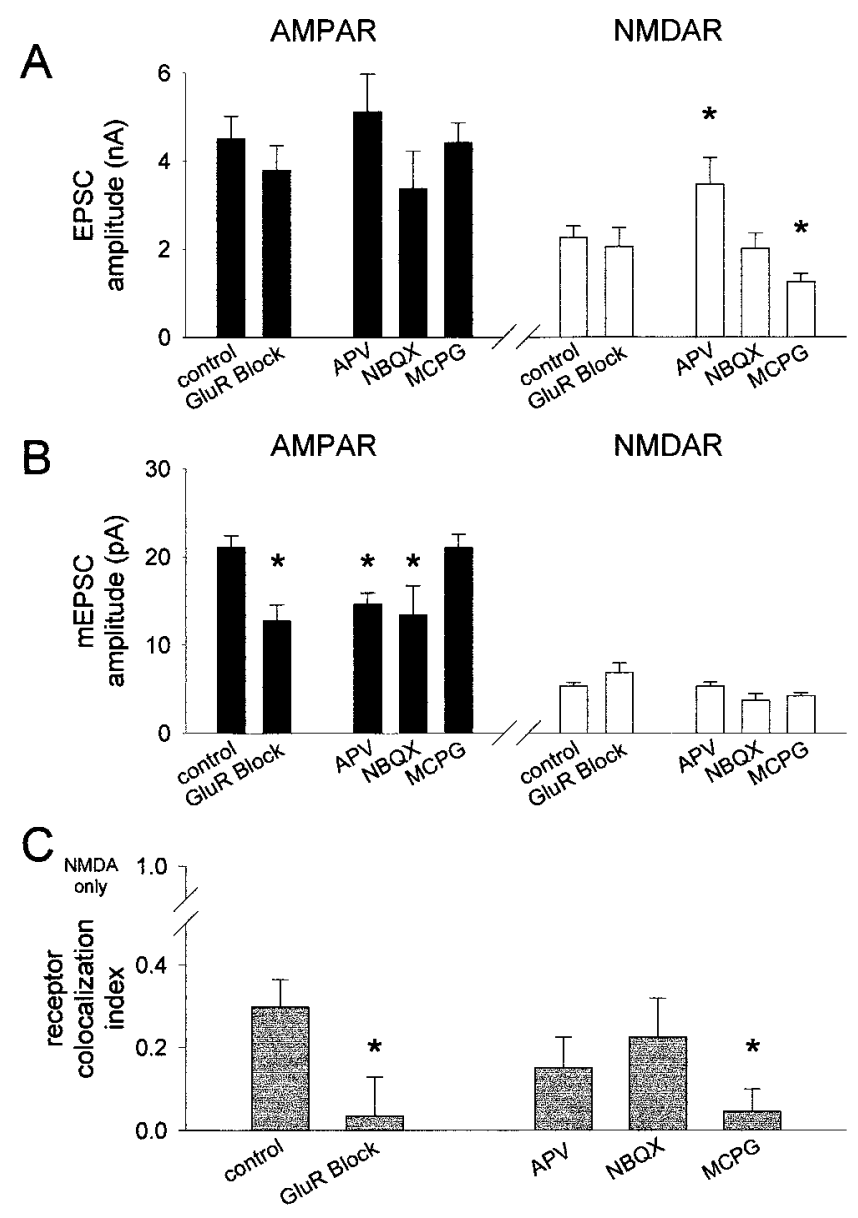

Figure 4. Chronic glutamate receptor blockade reduces both AMPAR quantal size and the fraction of NMDAR-only synapses. $A$, Evoked AMPAR and NMDAR EPSC amplitudes are unaffected by combined chronic glutamate receptor blockade with APV, NBQX, and MCPG but are differentially affected by APV, NBQX, and MCPG. No manipulation significantly affected AMPAR EPSC amplitude ( filled bars). APV significantly increased and MCPG significantly decreased NMDAR EPSC amplitude (open bars) (AMPAR and NMDAR EPSCs, control, $n=18$, 20; GluR blockade, $n=13,12$; APV, $n=12,12$; NBQX, $n=9$, 9; MCPG, $n=9,12$, respectively; NMDAR EPSCs, APV vs control, ${ }^{*} p<0.05$; MCPG vs control, $\left.{ }^{*} p<0.05\right)$. $B$, AMPAR quantal amplitude is reduced by ionotropic but not metabotropic glutamate receptor antagonists, but NMDAR quantal amplitude is unaffected. Glutamate receptor blockade, APV, and NBQX all significantly reduced AMPAR quantal size without affecting NMDAR quantal size (AMPAR and NMDAR mEPSCs, control, $n=18$, 13; GluR blockade, $n=8,8$; APV, $n=11,9$; NBQX, $n=$ 6, 6; MCPG, $n=10,10$, respectively; AMPAR mEPSCs, GluR blockade vs control, ${ }^{*} p<0.01$; APV vs control, ${ }^{*} p<0.01$; NBQX vs control, ${ }^{*} p<$ $0.05)$. $C$, The fraction of NMDAR-only synapses, estimated from samecell comparisons of evoked and spontaneous dual-component events, is reduced by glutamate receptor blockade and MCPG but not by APV or NBQX (control, $n=9$; GluR blockade, $n=7$; APV, $n=6$; NBQX, $n=$ 6; MCPG, $n=9$; GluR blockade vs control, ${ }^{*} p<0.05$; MCPG vs control, $\left.{ }^{*} p<0.01\right)$.

mediated EPSCs were still observed in cells grown in these conditions (see Fig. 4).

The influence of activity on synapse formation could alternatively be mediated by the action potential. For example, presynaptic (or postsynaptic) action potentials could drive the release of molecules other than glutamate that coordinate the formation of a synapse. To test whether action potentials are required for synapse formation, we grew neurons in the voltage-dependent $\mathrm{Na}$ channel antagonist TTX. As with total glutamate receptor blockade, TTX did not prevent or delay the onset of AMPAR and NMDAR EPSCs, which could be recorded by 3 div (see Fig. 6). These results demonstrate that formation of functional glutamatergic synapses can occur without glutamate receptor activation or action potentials.

\section{Testing a role for activity in synapse maturation}

Although neither synaptic nor cellular activity seems to be essential for synapse formation, activity could still be critical for the changes in synaptic properties that occur with synapse maturation. We therefore assessed a role for activity in the developmental changes that we observed, specifically the increases in AMPAR quantal size, in the total number of synapses, and in the proportion of synapses that have only NMDARs. Comparison of 11-14 div cells grown in combined ionotropic and metabotropic glutamate receptor antagonists with control cells of the same age supports a role for synaptic activity in the increase in AMPAR quantal size (Fig. 4B). The AMPAR mEPSC amplitude of cells grown under glutamate receptor blockade was $12.7 \pm 1.9 \mathrm{pA}(n=$ 8) compared with $21.1 \pm 1.3 \mathrm{pA}$ for control cells $(n=18 ; p<$ 0.01). Surprisingly, this reduction in AMPAR quantal amplitude was not associated with a reduction of evoked AMPAR EPSC amplitude (Fig. 4A). This discrepancy suggests that glutamate receptor blockade increases the number of synapses that have AMPARs.

The selective effect of glutamate receptor blockade on AMPAR quantal amplitude but not on evoked EPSC amplitude could originate either from a proliferation of synapses with AMPAR components or from a reduction in the fraction of NMDAR-only synapses. That the evoked and quantal NMDAR EPSC amplitudes were unaffected by incubation in glutamate receptor antagonists (treated, $n=12$ evoked; $n=8$ mEPSC; control, $n=20$ evoked; $n=13$ mEPSC) (Fig. $4 A, B$ ) suggests that the total number of synapses with NMDARs did not increase. To test whether combined ionotropic and metabotropic glutamate receptor blockade impacted the fraction of NMDAR-only synapses, we calculated the fraction of silent synapses from the disparity in AMPAR and NMDAR quantal contents. This analysis demonstrated a marked reduction in the proportion of NMDAR-only events of treated cells in comparison with control cells (treated, $n=7$; control, $n=9 ; p<0.05$ ) (Fig. $4 C$ ). We therefore conclude that total glutamate receptor blockade prevented the developmental increase in AMPAR quantal size and reduced the proportion of NMDAR-only synapses.

To test whether distinct classes of glutamate receptor underlie the effects of total glutamate receptor blockade on AMPAR quantal size and on the proportion of NMDAR-only synapses, we examined cells grown selectively in APV, NBQX, or MCPG. Cells grown in APV showed significant reductions in AMPAR quantal size compared with control, from $21.1 \pm 1.3$ to $14.6 \pm 1.3$ pA (control, $n=18$; APV, $n=11 ; p<0.01$ ) (Fig. $4 B$ ). NMDAR activation therefore seems to be necessary for the developmental increase in AMPAR quantal size. APV did not significantly affect evoked AMPAR EPSC amplitude $(n=12)$ (Fig. $4 A$ ), however, suggesting an increase in the AMPAR quantal content. In contrast to its actions on AMPAR quantal amplitude, APV did not affect NMDAR quantal amplitude, as measured from the dualcomponent mEPSC $(n=9)$ (Fig. $4 B)$. APV did, however, significantly increase the evoked NMDAR EPSC $(n=12 ; p<0.05)$ (Fig. $4 A$ ), consistent with an increase in the quantal content of the NMDAR response as well. 
The apparent increases in AMPAR and NMDAR quantal contents could derive from an increase in the probability of release or, alternatively, from an increase in the number of functional synapses. APV incubation did not change paired-pulse facilitation of the AMPAR EPSC (APV, paired pulse $=0.8 \pm$ $0.1 ; n=4$; control, paired pulse $=0.9 \pm 0.1 ; n=4$ ), however, suggesting that a change in the probability of release did not underlie the increases in quantal contents. APV is therefore likely to increase quantal content of both synaptic components by increasing the total number of synapses that neurons make. We next assessed whether NMDAR activation was responsible for the developmental increase in the proportion of NMDAR-only synapses. The calculated proportion of NMDAR-only synapses of APV-treated cells was not significantly different from control (APV, $n=6$ ) (Fig. 4C). Together, these data suggest that APV prevents the developmental increase in AMPAR quantal size and increases the total number of synapses, without detectably altering the proportion of NMDAR-only synapses.

Like APV, chronic AMPAR blockade with NBQX reduced AMPAR quantal size (NBQX, $n=6$; mean $=13.4 \pm 3.3 \mathrm{pA} ; p<$ 0.05). Both AMPAR and NMDAR activation thus appear to contribute to the developmental increase in AMPAR quantal amplitude. In contrast, NBQX incubation did not significantly affect the evoked AMPAR EPSC $(n=9)$ (Fig. 4A,B). This discrepancy suggests that NBQX may increase the number of synapses with AMPARs. NBQX did not significantly affect the evoked NMDAR EPSC $(n=9)$ or the quantal size of the NMDAR component of dual-component events $(n=6)$ (Fig. $4 A, B)$. The proportion of NMDAR-only synapses of cells grown in NBQX was also not significantly different from control (Fig. $4 C$ ). Therefore, chronic NBQX incubation selectively prevents only the developmental increase in AMPAR quantal size. Neither AMPAR nor NMDAR activation seems to be necessary for the activity-dependent developmental increase in the proportion of NMDAR-only synapses.

Having examined the effects of ionotropic glutamate receptor antagonists, we next turned to the effects of metabotropic glutamate receptor blockade using MCPG. Unlike APV and NBQX, MCPG incubation did not affect AMPAR quantal amplitude (MCPG, $n=10$ ) (Fig. 4B). Although MCPG also left evoked AMPAR EPSC amplitude unchanged $(n=9)$, the evoked NMDAR EPSC amplitude was significantly reduced $(n=12 ; p<$ 0.05) (Fig. 4A). Because the NMDAR component of dualcomponent mEPSCs was unaltered by MCPG treatment $(n=10)$ (Fig. 4B), MCPG selectively reduced the quantal content of the NMDAR synaptic component, consistent with a reduction in the proportion of NMDAR-only synapses. Indeed, the calculated proportion of NMDAR-only synapses was significantly reduced from control levels $(\mathrm{MCPG}$, mean $=0.04 \pm 0.05 ; n=9$; control, mean $=0.30 \pm 0.07 ; n=9 ; p<0.01)$ (Fig. $4 C$ ).

To obtain independent, nonelectrophysiological evidence of regulation of NMDAR-only synapses by metabotropic glutamate receptors, we examined the immunocytochemical staining of AMPARs (anti-GluR1) and NMDARs (anti-NR1) in 2-week-old autaptic cultures. The NR1 subunit is an essential component of all functional NMDARs, and the GluR1 subunit has been shown to colocalize precisely with the other AMPAR subunits (GluR2/3) that are expressed in hippocampal cultures (Craig et al., 1993). Figure $5 A$ shows the immunostaining of NR1 and GluR1 in representative isolated neurons. In untreated cells NR1 and GluR1 puncta colocalized extensively, but NR1 positive, GluR1 negative (NR1+/GluR1-) puncta were prevalent. Inter-
A

A
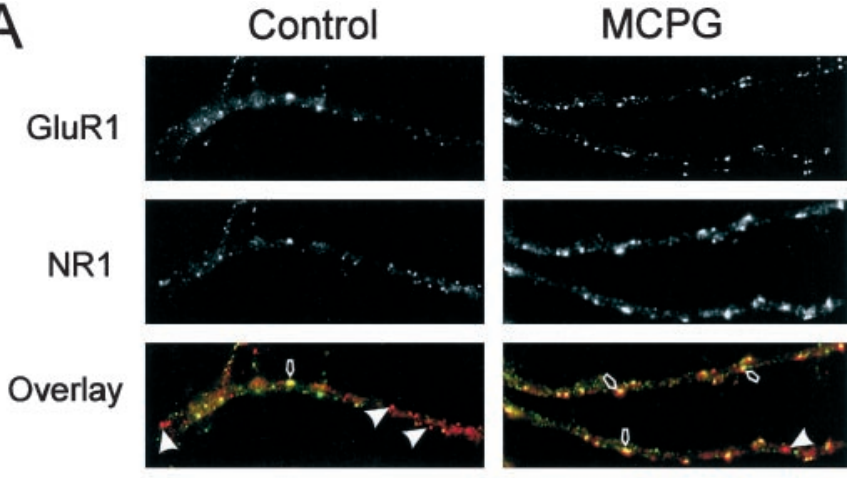

$B$

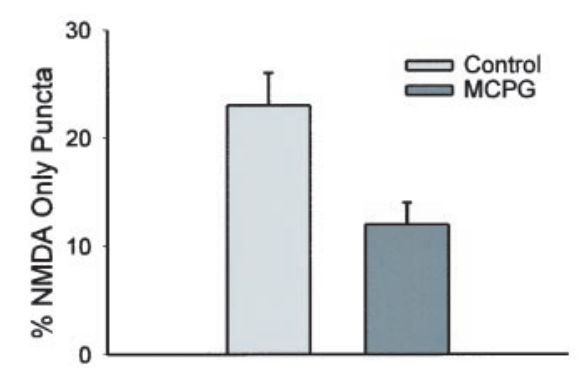

Figure 5. Chronic metabotropic glutamate receptor blockade reduces the proportion of NMDAR-only puncta. $A$, Images show immunocytochemical localization of GluR1 (top) and NR1(middle) in 2-week-old autaptic neurons grown in the absence (left) or presence (right) of the metabotropic glutamate receptor antagonist MCPG. Open arrows in overlayed images (bottom) identify colocalized NR1 and GluR1 puncta. White arrowheads identify examples of isolated NR1 puncta. B, Cells grown in MCPG had fewer NR1+/GluR1 - puncta than did cells grown in normal conditions $(p<0.01 ; n=31$ control cells, 26 MCPG-treated cells from three dissections).

estingly, chronic MCPG incubation significantly altered the pattern of NR1 and GluR1 staining, such that NR1+/GluR1puncta were much less frequently observed. As quantified in Figure $5 B, \mathrm{MCPG}$ treatment reduced the proportion of NR1+/ GluR1 - puncta by $\sim 47 \%$ (MCPG, $12.5 \pm 2 \%$; control, $23.5 \pm$ $3 \% ; p<0.01)$. In contrast, MCPG incubation had no effect on the proportion of GluR1 puncta not associated with NR1 puncta (GluR1+/NR1- puncta) (MCPG, $8.2 \pm 1 \%$; control, $8.6 \pm 1 \%$ ). These immunocytochemical results complement our physiological data and provide an anatomical correlate for the MCPGinduced reduction in the proportion of NMDAR-only synaptic responses. The effect of ionotropic and metabotropic glutamate receptor blockade on the maturation of synaptic function can therefore be dissected into two distinct pathways. Ionotropic receptor antagonists prevented the normal increase in AMPAR quantal size. Metabotropic glutamate receptor antagonists blocked the development of NMDAR-only synapses.

Because synaptic activity is greatly enhanced by presynaptic action potentials, we asked whether action potential blockade would impact synaptic maturation. The mean AMPAR EPSC $(n=8)$ and mean AMPAR quantal size $(n=8)$ of 2-week-old cells grown in TTX were significantly smaller than control $(p<$ 0.01 ), with quantal size similar to that of young neurons and of cells grown under glutamate receptor blockade (Fig. 6A,B). The reductions in both evoked and quantal components argue against a change in the AMPAR quantal content. It is interesting to note that the action of TTX was restricted to the AMPAR EPSC, because TTX did not affect the evoked NMDAR (TTX, $n=7$ ) 


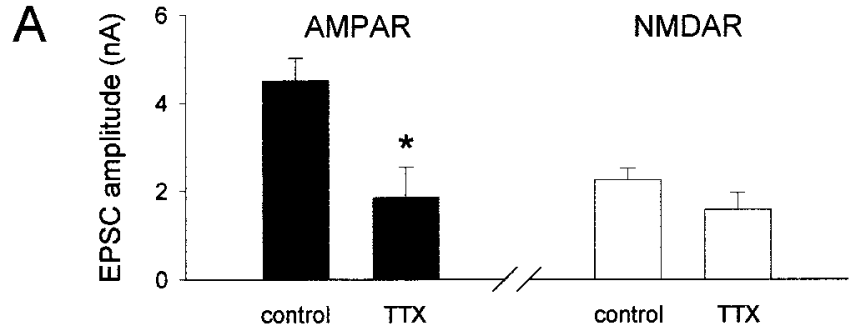

B
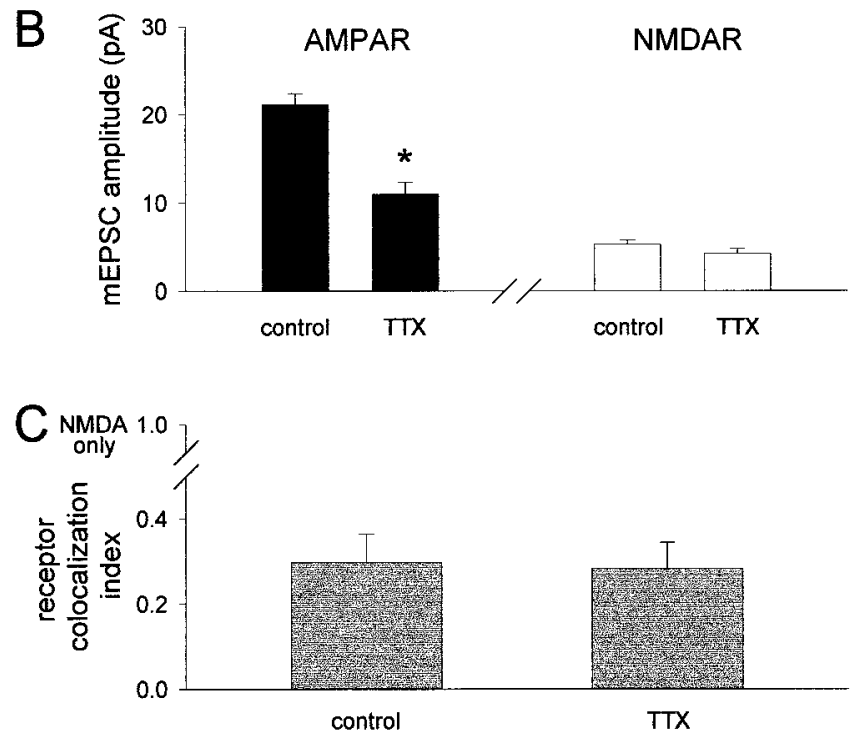

Figure 6. Chronic action potential blockade selectively interferes with the maturational increase in AMPAR quantal size. $A$, Evoked AMPAR EPSC amplitude but not NMDAR EPSC amplitude is reduced by chronic treatment with TTX. Filled bars represent AMPAR events; open bars represent NMDAR events (AMPAR and NMDAR EPSCs, control, $n=$ 18, 20; TTX, $n=8,7$, respectively; AMPAR EPSCs, TTX vs control, $* p<0.05)$. $B$, Mean AMPAR but not NMDAR quantal amplitude is reduced by TTX (AMPAR and NMDAR mEPSCs, control, $n=18,13$; TTX, $n=8,8$, respectively; AMPAR mEPSCs, TTX vs control, ${ }^{*} p<$ $0.01)$. $C$, The fraction of NMDAR-only synapses estimated from samecell comparisons of evoked and spontaneous dual-component events is not affected by TTX (control, $n=9$; TTX, $n=5$ ).

or the quantal NMDAR (TTX, $n=8$ ) EPSC (Fig. $6 A, B$ ). Furthermore, the proportion of NMDAR-only synapses of cells grown in TTX was identical to control (TTX, $n=5$ ) (Fig. $6 C$ ). These results suggest that action potentials contributed to the activity-dependent increase in AMPAR quantal size but not to the activity-dependent proliferation of NMDAR-only synapses.

To determine whether we could increase quantal size with a more effective action potential, we grew the neurons in the $\mathrm{K}$ channel blocker 4-AP at concentrations that markedly broaden the action potential ( $n=5$; data not shown). However, cells grown in 4-AP developed normal quantal (AMPAR, mean $=$ $16.7 \pm 2.3 \mathrm{pA} ; n=5 ;$ NMDAR, mean $=3.7 \pm 1.1 \mathrm{pA} ; n=5)$ and evoked (AMPAR, mean $=3.3 \pm 1.1 \mathrm{nA} ; n=5$; NMDAR, mean $=1.4 \pm 0.6 \mathrm{nA} ; n=5)$ AMPAR and NMDAR EPSC amplitudes. Our inability to alter synaptic properties by creating a more strongly depolarizing action potential waveform argues that a feature other than waveform, such as action potential frequency, is likely to underlie the role of action potentials in the increase in AMPAR quantal size during synapse maturation.

\section{DISCUSSION}

In this study we used single neurons grown in isolation as a model to study the formation and maturation of excitatory, glutamatergic synapses. This preparation benefits from the capacity to sample all synaptic inputs onto recorded cells over development, both with evoked and quantal synaptic transmission. In addition, it permits examination of NMDAR-only synaptic responses free of the possible contribution of spillover of glutamate to the observation of silent synapses (Gomperts et al., 1998). However, it should be kept in mind that growing neurons in isolation may alter processes involved in synapse maturation in the intact nervous system. We found that synapses continuously proliferated throughout our investigation. In fact, from the difference in the number of releasing synapses that we observed between young and old cells (and including cells that lacked responses), we estimate the rate of functional synapse formation to be approximately one synapse per hour.

Over this developmental period, AMPAR quantal size approximately doubled, while NMDAR quantal size was unaltered. One simple mechanism for the increase in mean AMPAR quantal size would be a developmental increase in quantal size at individual synapses. The developmental increase in the half-life of surface AMPARs that has been described (Mammen et al., 1997) could subserve this. However, the initial formation of synapses with small quantal size followed by the delayed formation of new synapses with large quantal size remains a plausible alternative. It should be noted that Liu and Tsien (1995) found no changes in AMPAR quantal size between 12 and 32 div in confluent hippocampal cultures. However, that study likely excluded the earliest time points of synapse development ( $<12 \mathrm{div})$.

After establishing the normal time course of synapse formation and maturation, we attempted to identify a role for synaptic and cellular activity specifically in synapse formation. Despite the blockade of all known glutamate receptors and despite action potential blockade, functional synapses formed with no detectable lag. This result clearly distinguishes glutamatergic synapses from spinal glycinergic synapses (Kirsch and Betz, 1998). It suggests that glutamatergic synapses may form in a manner resembling the neuromuscular junction, perhaps with the activityindependent release of an agrin-like molecule, such as NARP (O'Brien et al., 1999), coordinating the development of presynaptic and postsynaptic structures.

The autapse preparation does not appear to display the temporal profile of AMPAR and NMDAR EPSC development that has been observed in most other systems (Durand et al., 1996; Wu et al., 1996; Isaac et al., 1997; Hsia et al., 1998; Rumpel et al., 1998; Liao et al., 1999; but see Rao et al., 1998). Despite physiological and immunocytochemical evidence of NMDAR-only synapses in this preparation (Gomperts et al., 1998), we found no evidence of an NMDAR-only EPSC that precedes the development of AMPAR currents. However, because of the continuous synaptogenesis that occurs in this system, it is possible that at all but the earliest time points, cells express synapses of widely varying levels of maturity, making an NMDAR-only evoked EPSC difficult to observe.

A surprising finding was that a larger proportion of synapses are NMDAR-only in older cells. The developmental increase in the proportion of NMDAR-only synapses can be explained in three ways: (1) NMDAR-only synapses derive from synapses that originally had both receptor types, (2) NMDAR-only synapses are more stable than other synapses and are therefore selectively 
preserved, or (3) NMDAR-only synapse formation is selectively enhanced during development. The present data do not allow us to distinguish among these alternatives. The discrepancy in the developmental profile of NMDAR-only synaptic responses between this system and more intact preparations may be caused by differences in the developmental profile of metabotropic glutamate receptor activation (see below) or by differences in innervation density or trophic factor concentrations between preparations. Although the discrepancy could formally be attributable to a lack of contact with other neurons, the observation of a developmental increase in the proportion of NMDAR-only synapses agrees with immunocytochemical data from low-density confluent cultures (Rao et al., 1998; but see Liao et al., 1999).

Independent of the temporal profile of AMPAR and NMDAR EPSC development, NMDAR function in this preparation is clearly not required for the development of AMPAR-mediated synaptic transmission because NMDAR blockade did not impair the formation of AMPAR EPSCs. This result is consistent with immunocytochemical data that neurons cultured in APV and NBQX still form synaptic AMPAR puncta (O'Brien et al., 1997; Rao et al., 1998; Liao et al., 1999). Notably, the lack of effect of APV indicates that NMDAR-dependent forms of plasticity are not essential for the formation of AMPAR-containing synapses. This conclusion is consistent with observations in mice lacking the $\alpha$-calcium-calmodulin-dependent kinase II (Silva et al., 1992), in which AMPAR-mediated synaptic responses appeared normal despite a lack of NMDAR-dependent long-term potentiation.

It is interesting to note that even in the autapse preparation, a system without competition between neurons, activity sculpts synaptic responses. Although activity is not required for synapse formation, it does play an important role in the maturation of synaptic properties. Both ionotropic glutamate receptor blockade and action potential blockade prevented the developmental increase in AMPAR quantal size. One likely explanation of these data is that action potentials drive coordinated synaptic release of transmitter and the consequent postsynaptic depolarization, presumably via $\mathrm{Ca}$ influx, is required to increase quantal size. That APV alone prevented this increase suggests that $\mathrm{Ca}$ influx via the NMDAR may be particularly important in this maturational event. Another possibility is that the activity-dependent release of a growth factor such as BDNF, as has been shown previously in cortical cultures (Rutherford et al., 1998), could influence synapse maturation.

In contrast to AMPAR quantal size, NMDAR quantal size was insensitive to the pharmacological perturbations of activity. Differences in the association of NMDARs and AMPARs with structural elements at the synapse may underlie the differential capacity of AMPARs and NMDARs to be modified by activity. NMDARs are considerably more resistant to detergent extraction than are AMPARs (Allison et al., 1998). In addition, the depolymerization of filamentous actin spares NMDAR clusters but reduces the proportion of clustered AMPARs (Allison et al., 1998). Lastly, AMPARs and NMDARs interact with different PDZ-containing proteins at the synapse (Gomperts, 1996; Hsueh and Sheng, 1998; O'Brien et al., 1998). Taken together, these results suggest that the AMPAR is loosely coupled to the postsynaptic density whereas the NMDAR may be immobilized in the synaptic cytoskeleton.

Perhaps the most surprising result of this study was that the proportion of NMDAR-only synapses, assessed both physiologically and immunocytochemically, was significantly reduced when metabotropic glutamate receptors were blocked by MCPG yet this proportion was unaffected by TTX. This indicates that action potential-independent activation of metabotropic GluRs (mGluRs), presumably because of the spontaneous release of glutamate, plays a role in creating or maintaining the population of NMDAR-only synapses. This result is not the first indication that the spontaneous release of glutamate subserves an important function because such release also seems to be important for the maintenance of dendritic spines (McKinney et al., 1999; but see Kossel et al., 1997). In agreement with the effects of chronic MCPG exposure reported here, knock-out mice lacking mGluR5 also exhibited a reduction in the NMDAR/AMPAR EPSC amplitude ratio (Lu et al., 1997). Although our experiments do not explore the mechanism by which mGluRs influence the proportion of NMDAR-only synapses, one possibility is that they play a role analogous to that played by them in an mGluR-dependent form of long-term depression (LTD) in hippocampal slices (Oliet et al., 1997). This form of LTD is accompanied by a change in the frequency, but not the amplitude, of AMPAR mEPSCs, an observation consistent with the all-or-none downregulation of synaptic AMPAR clusters.

In contrast to previous immunocytochemical studies (Rao and Craig, 1997; Liao et al., 1999), we found that prolonged application of APV or CNQX failed to cause a detectable increase or decrease, respectively, in the proportion of NMDAR-only synapses. However, our inability to detect an effect of APV may stem from the maturity of the cells that we examined given the developmental increase in NMDAR-only synapses seen here and with immunocytochemistry (Rao et al., 1998). One important difference in the Liao et al. (1999) study is that drugs were applied to neurons with preexisting synapses rather than to neurons before synapse formation (see below). Differences between culture preparations or other experimental variables may also explain the discrepancy.

It is interesting that blockade of glutamate receptors prevented the developmental increase in AMPAR quantal size rather than increasing it, as has been reported previously for confluent cortical and hippocampal cultures (O'Brien et al., 1998; Turrigiano et al., 1998). The present study, however, examined the ability of a synapse to form and mature under a continuous manipulation of activity rather than the capacity of neurons with preexisting synaptic connections to regulate the amount of synaptic activation that they receive in response to an acute perturbation of activity. These results can be reconciled by proposing that neurons attempt to reach and maintain a set point for synaptic activation and upregulate or downregulate AMPAR quantal size to do so. We note that our effects of NBQX and TTX on the development of AMPAR quantal amplitude do resemble those seen in chick dissociated cultures (Kiyosue et al., 1996).

Our findings that activity does not impact the formation of glutamatergic synapses but does crucially regulate the final state of the synapse may have significant relevance to the activitydependent refinement of neural circuitry that occurs in vivo in a variety of systems (Katz and Shatz, 1996; Nguyen and Lichtman, 1996; Constantine-Paton and Cline, 1998). In principle, these changes could (but need not) occur in the context of activitydependent competition between synapses. In one such scenario, synapses driven by action potentials would activate their own complement of ionotropic and metabotropic receptors. Although postsynaptic depolarization would drive a homosynaptic potentiation, mGluR activation would produce a diffusible second messenger that would block AMPAR insertion or drive AMPAR 
removal from neighboring synapses. It will be interesting to explore these possibilities.

\section{REFERENCES}

Allen CN, Brady R, Swann J, Hori N, Carpenter DO (1988) N-MethylD-aspartate (NMDA) receptors are inactivated by trypsin. Brain Res 458:147-150.

Allison DW, Gelfand VI, Spector I, Craig AM (1998) Role of actin in anchoring postsynaptic receptors in cultured hippocampal neurons: differential attachment of NMDA versus AMPA receptors. J Neurosci 18:2423-2436.

Basarsky TA, Parpura V, Haydon PG (1994) Hippocampal synaptogenesis in cell culture: developmental time course of synapse formation, calcium influx, and synaptic protein distribution. J Neurosci 14:6402-6411.

Bekkers JM, Stevens CF (1989) NMDA and non-NMDA receptors are co-localized at individual excitatory synapses in cultured rat hippocampus. Nature 341:230-233.

Benson DL, Cohen PA (1996) Activity-independent segregation of excitatory and inhibitory synaptic terminals in cultured hippocampal neurons. J Neurosci 16:6424-6432.

Cohen MW (1972) The development of neuromuscular connexions in the presence of D-tubocurarine. Brain Res 41:457-463.

Constantine-Paton M, Cline HT (1998) LTP and activity-dependent synaptogenesis: the more alike they are, the more different they become. Curr Opin Neurobiol 8:139-148.

Craig AM, Blackstone CD, Huganir RL, Banker G (1993) The distribution of glutamate receptors in cultured rat hippocampal neurons: postsynaptic clustering of AMPA-selective subunits. Neuron 10:1055-1068.

Craig AM, Blackstone CD, Huganir RL, Banker G (1994) Selective clustering of glutamate and gamma-aminobutyric acid receptors opposite terminals releasing the corresponding neurotransmitters. Proc Natl Acad Sci USA 91:12373-12377.

Davey DF, Cohen MW (1986) Localization of acetylcholine receptors and cholinesterase on nerve-contacted and noncontacted muscle cells grown in the presence of agents that block action potentials. J Neurosci 6:673-680.

Durand GM, Kovalchuk Y, Konnerth A (1996) Long-term potentiation and functional synapse induction in developing hippocampus. Nature 381:71-75.

Gomperts SN (1996) Clustering membrane proteins: it's all coming together with the PSD-95/SAP90 protein family. Cell 84:659-662.

Gomperts SN, Rao A, Craig AM, Malenka RC, Nicoll RA (1998) Postsynaptically silent synapses in single neuron cultures. Neuron 21:1443-1451.

Hsia A, Malenka RC, Nicoll RA (1998) Development of excitatory circuitry in the hippocampus. J Neurophysiol 79:2013-2024.

Hsueh YP, Sheng M (1998) Anchoring of glutamate receptors at the synapse. Prog Brain Res 116:123-131.

Isaac JTR, Nicoll RA, Malenka RC (1995) Evidence for silent synapses: implications for the expression of LTP. Neuron 15:427-434.

Isaac JTR, Crair MC, Nicoll RA, Malenka RC (1997) Silent synapses during development of thalamocortical inputs. Neuron 18:269-280.

Katz LC, Shatz CJ (1996) Synaptic activity and the construction of cortical circuits. Science 274:1133-1138.

Kimura F, Otsu Y, Tsumoto T (1997) Presynaptically silent synapses: spontaneously active terminals without stimulus-evoked release demonstrated in cortical autapses. J Neurophysiol 77:2805-2815.

Kirsch J, Betz H (1998) Glycine-receptor activation is required for receptor clustering in spinal neurons. Nature 392:717-720.

Kiyosue K, Kasai M, Taguchi T (1996) Two modes of activitydependent synaptogenesis of cerebral neurons in vitro. NeuroReport 7:701-704

Kossel AH, Williams CV, Schweizer M, Kater SB (1997) Afferent innervation influences the development of dendritic branches and spines via both activity-dependent and nonactivity-dependent mechanisms. J Neurosci 17:6314-6324.

Lerma J, Morales M, Vicente MA, Herreras O (1997) Glutamate receptors of the kainate type and synaptic transmission. Trends Neurosci 20:9-12.

Liao D, Hessler NA, Malinow R (1995) Activation of postsynaptically silent synapses during pairing-induced LTP in CA1 region of hippocampal slice. Nature 375:400-404.

Liao D, Zhang X, O’Brien R, Ehlers MD, Huganir RL (1999) Regula- tion of morphological postsynaptic silent synapses in developing hippocampal neurons. Nat Neurosci 2:37-43.

Liu G, Tsien RW (1995) Properties of synaptic transmission at single hippocampal synaptic boutons. Nature 375:404-408.

Lu YM, Jia Z, Janus C, Henderson JT, Gerlai R, Wojtowicz JM, Roder JC (1997) Mice lacking metabotropic glutamate receptor 5 show impaired learning and reduced CA1 long-term potentiation (LTP) but normal CA3 LTP. J Neurosci 17:5196-5205.

Mammen AL, Huganir RL, O'Brien RJ (1997) Redistribution and stabilization of cell surface glutamate receptors during synapse formation. J Neurosci 17:7351-7358.

Manabe T, Wyllie DJA, Perkel DJ, Nicoll RA (1993) Modulation of synaptic transmission and long-term potentiation: effects on paired pulse facilitation and EPSC variance in the CA1 region of the hippocampus. J Neurophysiol 70:1451-1459.

McKinney RA, Capogna M, Durr R, Gahwiler BH, Thompson SM (1999) Miniature synaptic events maintain dendritic spines via AMPA receptor activation. Nat Neurosci 2:44-49.

Murase K, Ryu PD, Randic M (1989) Excitatory and inhibitory amino acids and peptide-induced responses in acutely isolated rat spinal dorsal horn neurons. Neurosci Lett 103:56-63.

Nguyen QT, Lichtman JW (1996) Mechanism of synapse disassembly at the developing neuromuscular junction. Curr Opin Neurobiol 6:104-112.

Nusser Z, Lujan R, Laube G, Roberts JDB, Molar E, Somogyi P (1998) Cell type and pathway dependence of synaptic AMPA receptor number and variability in the hippocampus. Neuron 21:545-559.

O'Brien RJ, Mammen AL, Blackshaw S, Ehlers MD, Rothstein JD, Huganir RL (1997) The development of excitatory synapses in cultured spinal neurons. J Neurosci 17:7339-7350.

O'Brien RJ, Lau LF, Huganir RL (1998) Molecular mechanisms of glutamate receptor clustering at excitatory synapses. Curr Opin Neurobiol 8:364-369.

O’Brien RJ, Xu D, Petralia RS, Steward O, Huganir RL, Worley P (1999) Synaptic clustering of AMPA receptors by the extracellular immediateearly gene product NARP. Neuron 23:309-323.

Oliet SHR, Malenka RC, Nicoll RA (1997) Two distinct forms of longterm depression coexist in CA1 hippocampal pyramidal cells. Neuron 18:969-982.

Petralia RS, Esteban JA, Wang YX, Partridge JG, Zhao HM, Wenthold RJ, Malinow R (1999) Selective acquisition of AMPA receptors over postnatal development suggests a molecular basis for silent synapses. Nat Neurosci 2:31-36.

Rao A, Craig AM (1997) Activity regulates the synaptic localization of the NMDA receptor in hippocampal neurons. Neuron 19:801-812.

Rao A, Kim E, Sheng M, Craig AM (1998) Heterogeneity in the molecular composition of excitatory postsynaptic sites during development of hippocampal neurons in culture. J Neurosci 18:1217-1229.

Rumpel S, Hatt H, Gottmann K (1998) Silent synapses in the developing rat visual cortex: evidence for postsynaptic expression of synaptic plasticity. J Neurosci 18:8863-8874.

Rutherford LC, Nelson SB, Turrigiano GG (1998) BDNF has opposite effects on the quantal amplitude of pyramidal neuron and interneuron excitatory synapses. Neuron 21:521-530.

Segal MM, Furshpan EJ (1990) Epileptiform activity in microcultures containing small numbers of hippocampal neurons. J Neurophysiol 64:1390-1399.

Silva AJ, Stevens CF, Tonegawa S, Wang Y (1992) Deficient hippocampal long-term potentiation in alpha calcium-calmodulin kinase II mutant mice. Science 257:201-206.

Steward O, Falk PM (1991) Selective localization of polyribosomes beneath developing synapses: a quantitative analysis of the relationships between polyribosomes and developing synapses in the hippocampus and dentate gyrus. J Comp Neurol 314:545-557.

Takumi Y, Ramirez-Leon V, Laake P, Rinvik E, Ottersen OP (1999) Different modes of expression of AMPA and NMDA receptors in hippocampal synapses. Nat Neurosci 2:618-624.

Tong G, Jahr CE (1994) Block of glutamate transporters potentiates postsynaptic excitation. Neuron 13:1195-1203.

Turrigiano GG, Leslie KR, Desai NS, Rutherford LC, Nelson SB (1998) Activity-dependent scaling of quantal amplitude in neocortical neurons. Nature 391:892-896.

Wu G, Malinow R, Cline HT (1996) Maturation of a central glutamatergic synapse. Science 274:972-976. 